Pacific Journal of Mathematics

CONDITIONAL SCHAUDER DECOMPOSITIONS OF
NORMED IDEALS OF OPERATORS BETWEEN SOME
$l_{p}$-SPACES 


\section{UNCONDITIONAL SCHAUDER DECOMPOSITIONS OF NORMED IDEALS OF OPERATORS BETWEEN SOME $l_{p}$-SPACES}

\section{Y. GORDON}

Given a Banach space $E$, let

$$
l(E)=\sup _{F \in \mathscr{T}(E)} \inf _{\left\{P_{i}\right\}} \sup _{N, \pm}\left\|\sum_{i=1}^{N} \pm \sqrt{r\left(P_{i}\right)} P_{i}\right\|
$$

where $\mathscr{F}(E)$ denotes the collection of all finite-dimensional subspaces of $E$, the infimum ranges over all possible sequences of finite-rank operators $P_{i}: F \rightarrow E$ which satisfy the equality $\Sigma P_{i}(f)=f$ for all $f \in F$, and $r(P)$ denotes the rank of an operator $P$.

It is shown that there are finite-dimensional spaces with arbitrarily large $l(E)$ values, and infinite-dimensional spaces $E$ with $l(E)=\infty$. The specific examples with $l(E)=\infty$ yield also information on the rapidity of growth of unconditional Schauder decompositions of $E$ into finite-dimensional spaces.

Clearly if $E$ is finite-dimensional

$$
l(E)=\inf _{\left\{P_{i}\right\}} \sup _{N, \pm}\left\|\sum_{i=1}^{N} \pm \sqrt{r\left(P_{i}\right)} P_{\imath}\right\|
$$

where the infimum ranges over all sequences $P_{i}: E \rightarrow E$ satisfying $\sum_{i \geqq 1} P_{i}(x)=x$ for all $x \in E$.

It is also obvious from the definition that the value $l(E)$ is not greater than the local unconditional constant $\chi_{u}(E)$ introduced in [3] which is defined similarly, the only difference being that for $\chi_{u}(E)$ only sequences $\left\{P_{i}\right\}$ with $r\left(P_{i}\right)=1$ for all $i$ are considered. Spaces $E$ with finite $\chi_{u}(E)$ were called in [3] spaces with local unconditional structure. If $E$ is complemented in a space with an unconditional basis then clearly $\chi_{u}(E)<\infty$.

Besides this generalization the result stated above answers a question of Professor H. P. Rosenthal by providing examples of spaces which do not have unconditional Schauder decompositions into finitedimensional spaces all of the same dimension $p$, for any $p=1,2,3, \cdots$; spaces $E$ with $l(E)=\infty$ clearly cannot have such decompositions.

Specifically it is shown in section 2 that if $E$ is the space of operators on $l_{2}$ equipped with any ideal norm $\alpha$, then $l(E)=\infty$ unless $\alpha$ is 
equivalent to the Hilbert-Schmidt norm for operators on $l_{2}$. This implies Lewis' ([6]) characterization of the ideals of operators on $l_{2}$ which have local unconditional structure. In addition, it is proved in section 3 that the space $E$ of operators mapping $l_{1}$ to $c_{0}$ normed with any perfect ideal norm $\alpha$ which is not equivalent to the operator norm $\|\circ\|$, also has $l(E)=\infty$. Additional results on spaces with $l(E)=\infty$ will appear in a forthcoming paper by Professor P. Saphar and this author.

If $l(E)=\infty$, then by Proposition $1 E$ does not have property $P_{k}$ for any integer $k=1,2, \cdots$; according to Lindenstrauss and Zippin ([7]) a Banach space $E$ has property $P_{k}$ if there is a $\lambda>0$ such that for every finite-dimensional subspace $F$ of $E$ there is a Boolean algebra of projections $\mathscr{B}$ on $E$ with $\sup \{\|P\| ; P \in \mathscr{B}\} \leqq \lambda$ and $k$ vectors $\left\{x_{i}\right\}_{1}^{k}$ in $E$ such that $F$ is contained in the closed linear span of $\left\{P\left(x_{i}\right) ; i=1, \cdots, k\right.$, $P \in \mathscr{B}\}$.

The terminology will generally follow that of [4]. $\mathscr{R}^{n}$ will denote the $n$-dimensional linear space, $\left\{e_{i}\right\}_{1}^{n}$ the usual unit basis. Given any vector $\epsilon=\left(\epsilon_{1}, \epsilon_{2}, \cdots, \epsilon_{n}\right)$ with $\epsilon_{i}= \pm 1, h_{\epsilon}$ will denote the linear operator on $\mathscr{R}^{n}$ defined by $h_{\epsilon}\left(e_{i}\right)=\epsilon_{i} e_{i}$ for all $i$. For any permutation $\sigma$ of $\{1,2, \cdots, n\}, g_{\sigma}$ will denote the operator defined by $g_{\sigma}\left(e_{\imath}\right)=e_{\sigma(i)}$ for all $i$.

$G$ will be the compact group of all isometries on $l_{2}^{n}$ and $d g$ its unique normalized Haar measure. $S$ will be the unit sphere in $l_{2}^{n}\{x \in$ $\left.l_{2}^{n} ;\|x\|_{2}=1\right\}$ and $d x$ will stand for the probability measure on $S$ defined by

$$
\int_{S} f(x) d x=\int_{G} f(g(e)) d g, \quad f \in C(S)
$$

where $e \in S$ is any fixed point.

Given any ideal norm $\alpha([4])$ and a Banach space $E, \alpha(E)$ will stand for the value $\alpha\left(1_{E}\right)$ where $1_{E}$ is the identity operator on $E$. $\alpha^{*}$ will denote the adjoint ideal norm of $\alpha$. $\alpha$ is perfect if $\alpha^{* *}=$ $\alpha$. $[L(E, F), \alpha]$ will be the space of all operators $T: E \rightarrow F$ with $\alpha(T)<\infty$, and $E^{\prime}$ denotes the conjugate of $E$.

Recall that if $E$ and $F$ are finite-dimensional $[L(E, F), \alpha]^{\prime}$ is the space $\left[L(F, E),, \alpha^{*}\right]$ where the correspondence is given by

$$
\langle S, T\rangle=\operatorname{trace}(S T), \quad S \in L(E, F), \quad T \in L(F, E) .
$$

$\pi_{p}$ and $i_{p}(1 \leqq p \leqq \infty)$ will denote the $p$-absolutely summing and $p$ integral norms respectively. All Banach spaces are taken over the reals as the results can be easily carried over to the complex case with some changes in the constants.

Lemma 1. If $A \in L(X, E), B \in L(E, X)$ and $B A$ is the identity on $X$, then $l(X) \leqq\|A\|\|B\| l(E)$. 
Proof. Let $F \in \mathscr{F}(X)$ and $\epsilon>0$ be given. $\quad A(F) \in \mathscr{F}(E)$, so there are $P_{\imath}: A(F) \rightarrow E$ with $\Sigma P_{i} A(f)=A(f)$ for all $f \in F$ and

$$
\sup _{ \pm, N}\left\|\sum_{1}^{N} \pm \sqrt{r\left(P_{i}\right)} P_{i}\right\| \leqq l(E)+\epsilon .
$$

Set $Q_{\imath}=B P_{i} A$, then $r\left(Q_{\imath}\right) \leqq r\left(P_{i}\right)$ and $\Sigma Q_{i}(f)=f$ for all $f \in F$, and

$$
\sup _{ \pm, N}\left\|\sum_{1}^{N} \pm \sqrt{r\left(Q_{i}\right)} Q_{i}\right\| \leqq\|A\|\|B\|(l(E)+\epsilon) .
$$

As $\epsilon$ and $F$ are arbitrary, the result follows.

Proposition 1. If $E$ has property $P_{k}$ for some integer $k$, then $l(E)<\infty$.

Proof. There is a $\lambda>0$ such that if $F \subset E$ is any finite-dimensional subspace there is a subset $\left\{x_{i}\right\}_{1}^{k} \subset E$ and a Boolean algebra of projections $\mathscr{B}$ on $E$, with $\sup \{\|P\| ; P \in \mathscr{B}\} \leqq \lambda$ and $F \subset \operatorname{span}\left\{P\left(x_{j}\right) ; j=1, \cdots, k\right.$, $P \in \mathscr{B}\}$.

Using elementary arguments similar to Proposition 1 of [7], let $\left\{y_{1}, \cdots, y_{p}\right\}$ be a basis of $F$. Given $\epsilon$, there exists a subset of $n$-disjoint elements $\left\{P_{i}\right\}_{1}^{n} \subset \mathscr{B} \quad$ with $\sum_{1}^{n} P_{i}=I$, a subset $\left\{z_{r}\right\}_{1}^{p} \subset \operatorname{span}\left\{P_{1}\left(x_{j}\right)\right.$; $i=1, \cdots, n, j=1, \cdots, k\}$ with $\left\|z_{r}-y_{r}\right\|<\epsilon$ for every $r=1, \cdots, p$. It is easy to see that if $\epsilon>0$ is sufficiently small there is a $1-1$ operator $T$ on $E$ satisfying $T\left(y_{r}\right)=z_{r}$ for all $r$ and $\|T\|,\left\|T^{-1}\right\|<2$.

Let $R_{i}$ be the restriction to $F$ of $T^{-1} P_{i} T, i=1, \cdots, n$. Then $r\left(R_{i}\right) \leqq k, \sum R_{\imath}$ is the identity on $F$ and

$$
\begin{aligned}
\sup _{ \pm}\left\|\sum_{1}^{n} \pm \sqrt{r\left(R_{i}\right)} R_{i}\right\| & \leqq \sqrt{k} \sup _{ \pm}\left\|\sum_{1}^{n} \pm T^{-1} P_{i} T\right\| \\
& \leqq 4 \sqrt{k} \sup _{ \pm}\left\|\sum_{1}^{n} \pm P_{i}\right\| \leqq 8 \sqrt{k} \sup _{J}\left\|\sum_{i \in J} P_{i}\right\| \\
& \leqq 8 \sqrt{k} \lambda,
\end{aligned}
$$

this proves $l(E) \leqq 8 \sqrt{k} \lambda$.

The following elementary generalization of Hölder's inequality will be used.

Lemma 2. Let $x_{k}, y_{k} k=1,2, \cdots, n$ be vectors in $\mathscr{R}^{m}$. Then 


$$
\left(\sum_{k=1}^{n}\left\langle x_{k}, y_{k}\right\rangle\right)^{2} \leqq m \sum_{j=1}^{n} \sum_{k=1}^{n}\left\langle x_{k}, y_{j}\right\rangle^{2}
$$

Proof. Assume without loss of generality that $\operatorname{span}\left\{y_{k}\right\}=\mathscr{R}^{m}$. Fix the sequence $\left\{y_{k}\right\}$ and consider the problem of minimizing the function

$$
f\left(\left\{x_{k}\right\}\right)=\sum_{j=1}^{n} \sum_{k=1}^{n}\left\langle x_{k}, y_{j}\right\rangle^{2} \text { under the restriction } \sum_{k=1}^{n}\left\langle x_{k}, y_{k}\right\rangle=1
$$

Using the "Lagrange multipliers" method, set

$$
\phi\left(\left\{x_{k}\right\}\right)=\sum_{j=1}^{n} \sum_{k=1}^{n}\left\langle x_{k}, y_{j}\right\rangle^{2}-\lambda\left(\sum_{k=1}^{n}\left\langle x_{k}, y_{k}\right\rangle\right) \text {. }
$$

At the minimum value for $f$, which must exist, $\partial \phi / \partial x_{k t}=0$ for all $k=1,2, \cdots, n, i=1,2, \cdots, m$, where $x_{k}=\sum_{i=1}^{m} x_{k i} e_{i}$. The equations in vector form are then

$$
\sum_{j=1}^{n}\left\langle x_{k}, y_{j}\right\rangle y_{j}=\lambda y_{k} \text { for all } k=1,2, \cdots, n
$$

Clearly $\lambda \neq 0$, so the operator $A=\sum_{j=1}^{n} y_{l} \otimes y_{j}$ satisfies the equations $A\left(x_{k}\right)=\lambda y_{k}$ for all $k$, hence has an inverse $A^{-1}$. Then

$$
\begin{aligned}
m & =\operatorname{trace}\left(A^{-1} A\right)=\sum_{j=1}^{n}\left\langle y_{j}, A^{-1}\left(y_{j}\right)\right\rangle=\sum_{j=1}^{n} \lambda^{-1}\left\langle y_{j}, x_{j}\right\rangle \\
& =\lambda^{-1}
\end{aligned}
$$

finally,

$$
f\left(\left\{x_{k}\right\}\right)=\sum_{k=1}^{n} \sum_{j=1}^{n}\left\langle x_{k}, y_{j}\right\rangle^{2}=\sum_{k=1}^{n}\left\langle\lambda y_{k}, x_{k}\right\rangle=\lambda=m^{-1}
$$

2. Unconditional decomposition of ideals of operators between Hilbert spaces. The main result proved here is the following:

Theorem 1. Let $\alpha$ be an ideal norm, $E=\left[L\left(l_{2}^{n}, l_{2}^{n}\right), \alpha\right]$, and let $\alpha(n)=\max \left\{\max \left\{\alpha(A) / \pi_{2}(A), \pi_{2}(A) / \alpha(A)\right\} ; A \in L\left(l_{2}^{n}, l_{2}^{n}\right)\right\}$. Then,

$$
e^{1 / 2}(\pi / 2)^{2} l(E) \geqq \alpha(n) .
$$

Proof. Let $u=\sum_{i=1}^{m} A_{i} \otimes B_{i}$ be any rank- $m$ operator mapping $E$ to $E$, where $A_{i} \in E^{\prime}=\left[L\left(l_{2}^{n}, l_{2}^{n}\right), \alpha^{*}\right]$ and $B_{i} \in E$. We shall write 


$$
A_{i}\left(e_{j}\right)=\sum_{k=1}^{n} a_{i j k} e_{k} \quad \text { and } \quad B_{i}\left(e_{j}\right)=\sum_{k=1}^{n} b_{i j k} e_{k}
$$

for all $i=1, \cdots, m, j=1, \cdots, n$, where $\left\{e_{k}\right\}_{1}^{n}$ is the unit basis of $l_{2}^{n}$. Denote by $K_{F}$ the unit ball of a given Banach space $F$, and let $K=K_{\epsilon} \times K_{E^{\prime}}$ be the product of the unit balls. Define on $K$ the probability measure $\mu$ by

$$
\mu(f)=\int_{G} \int_{G} 2^{-n} \sum_{\epsilon} \int_{S} \int_{S} f\left(\left(\left[\alpha(A)^{-1} h_{\epsilon} g A h\right) \times(y \otimes x)\right) d y d x d g d h\right.
$$

where $f \in C(K), A \in L\left(l_{2}^{n}, l_{2}^{n}\right)$ is a fixed non-zero operator, and $\Sigma_{\epsilon}$ denotes the sum over all $2^{n}$ possible choices of $\epsilon=( \pm 1, \pm 1, \cdots, \pm 1)$.

The operator $u$ defines a function of $C(K)$ which is denoted by $\langle u, \circ\rangle$ and defined as $\langle u, a \times b\rangle=\langle u(a), b\rangle=\operatorname{trace}(b(u(a))), a \in E, b \in E^{\prime}$.

Then,

$$
\begin{aligned}
\alpha(A) \mu & (|\langle u, \circ\rangle|) \\
& =\int_{G} \int_{G} 2^{-n} \sum_{\epsilon} \int_{S} \int_{S}\left|\left\langle y \otimes x, u\left(h_{\epsilon} g A h\right)\right\rangle\right| d y d x d g d h \\
& =\int_{G} \int_{G} 2^{-n} \sum_{\epsilon} \int_{S} \int_{S}\left|\left\langle\left(u\left(h_{\epsilon} g A h\right)\right)(x), y\right\rangle\right| d y d x d g d h .
\end{aligned}
$$

It is well known ([1]) that for any $v \in L\left(l_{2}^{n}, l_{2}^{n}\right)$ with $v_{j k}=\left\langle v\left(e_{l}\right), e_{k}\right\rangle$

$$
\left(\pi_{1}\left(l_{2}^{n}\right)\right)^{2} \int_{S} \int_{S}|\langle v(x), y\rangle| d y d x=\pi_{1}(v) \geqq \pi_{2}(v)=\left(\sum_{j, k=1}^{n} v_{j k}^{2}\right)^{1 / 2}
$$

therefore

$$
\begin{aligned}
& \left(\pi_{1}\left(l_{2}^{n}\right)\right)^{2} 2^{-n} \sum_{\epsilon} \int_{S} \int_{S}\left|\left\langle\left(u\left(h_{\epsilon} g A h\right)\right)(x), y\right\rangle\right| d y d x \\
& \geqq 2^{-n} \sum_{\epsilon} \pi_{2}\left(\sum_{i=1}^{m}\left(\operatorname{trace}\left(h_{\epsilon} g A h A_{i}\right)\right) B_{i}\right) \\
& =2^{-n} \sum_{\epsilon}\left[\sum_{j, k=1}^{n}\left(\operatorname{trace}\left\{h_{\epsilon} g A h\left(\sum_{i=1}^{m} b_{i j k} A_{i}\right)\right\}\right)^{2}\right]^{1 / 2} .
\end{aligned}
$$

It is well known and easy to show that if $(\Omega, \Sigma, \mu)$ is a probability space and $f_{i} \in C(\Omega) i=1, \cdots, k$, then

$$
\mu\left(\left[\sum_{1}^{n}\left|f_{i}\right|^{2}\right]^{1 / 2}\right) \geqq\left[\sum_{1}^{n}\left(\mu\left(\left|f_{i}\right|\right)\right)^{2}\right]^{1 / 2}
$$


therefore

$$
\begin{aligned}
& \left(\pi_{1}\left(l_{2}^{n}\right)\right)^{2} 2^{-n} \sum_{\epsilon} \int_{S} \int_{S}\left|\left\langle\left(u\left(h_{\epsilon} g A h\right)\right)(x), y\right\rangle\right| d y d x \\
& \geqq\left[\sum_{,, k=1}^{n}\left(2^{-n} \sum_{\epsilon}\left|\operatorname{trace}\left\{h_{\epsilon} g A h\left(\sum_{i=1}^{m} b_{i j k} A_{i}\right)\right\}\right|\right)^{2}\right]^{1 / 2} \\
& \geqq e^{-1 / 2}\left[\sum_{s, j, k=1}^{n}\left\langle g A h\left(\sum_{i=1}^{m} b_{i j k} A_{i}\right)\left(e_{s}\right), e_{s}\right\rangle^{2}\right]^{1 / 2}
\end{aligned}
$$

the last is Khinchin's inequality (the constant $e^{-1 / 2}$ is due to [9]). Thus

$$
\begin{aligned}
\alpha(A) & \left(\pi_{1}\left(l_{2}^{n}\right)\right)^{2} e^{1 / 2} \mu(|\langle u, \circ\rangle|) \\
& \geqq \int_{G} \int_{G}\left[\sum_{s, j, k=1}^{n}\left\langle g A h\left(\sum_{l=1}^{m} b_{i j k} A_{i}\right)\left(e_{s}\right), e_{s}\right\rangle^{2}\right]^{1 / 2} \\
& \geqq\left[\sum_{s, l, k=1}^{n}\left(\int_{G} \int_{G}\left|\left\langle g A h\left(\sum_{l=1}^{m} b_{i l k} A_{i}\right)\left(e_{s}\right), e_{s}\right\rangle\right| d g d h\right)^{2}\right]^{1 / 2} \\
& =\left[\sum_{s, j, k=1}^{n}\left(\int_{G}\left\|A h\left(\sum_{i=1}^{m} b_{i j k} A_{i}\right)\left(e_{s}\right)\right\|_{2}\left(\pi_{1}\left(l_{2}^{n}\right)\right)^{-1} d h\right)^{2}\right]^{1 / 2} .
\end{aligned}
$$

Set $w=\sum_{i=1}^{m} b_{i j k} A_{\imath}$, then

$$
\begin{aligned}
\int_{G} \| & A h w\left(e_{s}\right) \|_{2} d h \\
& =\int_{G}\left(\sum_{t=1}^{n}\left\langle A h w\left(e_{s}\right), e_{t}\right\rangle^{2}\right)^{1 / 2} d h \\
& \geqq\left[\sum_{t=1}^{n}\left(\int_{G}\left|\left\langle A h w\left(e_{s}\right), e_{t}\right\rangle\right| d h\right)^{2}\right]^{1 / 2} \\
& =\left[\sum_{t=1}^{n}\left\|A^{\prime}\left(e_{t}\right)\right\|_{2}^{2}\left\|w\left(e_{s}\right)\right\|_{2}^{2}\right]^{1 / 2}\left(\pi_{1}\left(l_{2}^{n}\right)\right)^{-1},
\end{aligned}
$$

this implies

$$
\begin{aligned}
& \alpha(A) e^{1 / 2}\left(\pi_{1}\left(l_{2}^{n}\right)\right)^{4} \mu(|\langle u, \circ\rangle|) \\
& \geqq\left[\sum_{j, k, s, t=1}^{n}\left\|A^{\prime}\left(e_{t}\right)\right\|_{2}^{2}\left\|w\left(e_{s}\right)\right\|_{2}^{2}\right]^{1 / 2} \\
& =\pi_{2}(A)\left(\sum_{j, k, s, r=1}^{n}\left(\sum_{i=1}^{m} b_{i j k} a_{i s r}\right)^{2}\right)^{1 / 2} \\
& \geqq \pi_{2}(A)\left|\sum_{j, k=1}^{n} \sum_{i=1}^{m} b_{i j k} a_{i k j}\right| m^{-1 / 2} \\
& =\pi_{2}(A) m^{-1 / 2}|\operatorname{trace}(u)| .
\end{aligned}
$$


Let now $P_{\imath} \in L(E, E), i=1,2, \cdots, N$. Then

$$
\begin{gathered}
e^{1 / 2}\left(\pi_{1}\left(l_{2}^{n}\right)\right)^{4} \max _{ \pm}\left\|\sum_{i=1}^{N} \pm \sqrt{r\left(P_{i}\right)} P_{i}\right\| \\
\geqq e^{1 / 2}\left(\pi_{1}\left(l_{2}^{n}\right)\right)^{4} \mu\left(\sum_{i=1}^{N} \sqrt{r\left(P_{i}\right)}\left|\left\langle P_{i}, \circ\right\rangle\right|\right) \\
\geqq\left(\pi_{2}(A) / \alpha(A)\right)\left|\sum_{i=1}^{N} \operatorname{trace}\left(P_{i}\right)\right|
\end{gathered}
$$

As $\alpha$ and $P_{i}$ are arbitrary, the inequality is true for $\alpha^{*}$ and $P_{1}^{\prime}$ too, noting that $P_{i}^{\prime}$ maps $\left[L\left(l_{2}^{n}, l_{2}^{n}\right), \alpha^{*}\right]$ to itself, and as

$$
\begin{aligned}
\sum_{1}^{N} \operatorname{trace}\left(P_{i}^{\prime}\right) & =\sum_{1}^{N} \operatorname{trace}\left(P_{i}\right) \text { and }\left\|\sum_{1}^{N} \pm \sqrt{r\left(P_{i}^{\prime}\right)} P_{i}^{\prime}\right\| \\
& =\left\|\sum_{1}^{N} \pm \sqrt{r\left(P_{i}\right)} P_{i}\right\|,
\end{aligned}
$$

it follows for arbitrary non-zero operators $A, B$ on $l_{2}^{n}$ that

$$
\begin{gathered}
e^{1 / 2}\left(\pi_{1}\left(l_{2}^{n}\right)\right)^{4} \max _{ \pm}\left\|\sum_{1}^{N} \pm \sqrt{r\left(P_{i}\right)} P_{i}\right\| \\
\geqq \max \left\{\pi_{2}(A) / \alpha(A), \pi_{2}(B) / \alpha^{*}(B)\right\}\left|\operatorname{trace}\left(\sum_{1}^{N} P_{i}\right)\right| .
\end{gathered}
$$

Finally, if $\sum_{1 \geqq 1} P_{i}(x)=x$ for all $x \in E$, then trace $\left(\sum_{i \geqq 1} P_{i}\right)=n^{2}$ and the result follows from the inequality $\pi_{1}\left(l_{2}^{n}\right) \leqq \sqrt{\pi n / 2}$ ([2]).

COROllary 1. If $\alpha$ is not equivalent to the Hilbert-Schmidt norm for operators on $l_{2}$, then

$$
l\left(\left[L\left(l_{2}^{n}, l_{2}^{n}\right), \alpha\right]\right) \underset{n \rightarrow \infty}{\longrightarrow} \infty \quad \text { and } \quad l\left(\left[L\left(l_{2}, l_{2}\right), \alpha\right]\right)=\infty
$$

Proof. Let $J_{n}:\left[L\left(l_{2}^{n}, l_{2}^{n}\right), \alpha\right] \rightarrow\left[L\left(l_{2}, l_{2}\right), \alpha\right]$ be the natural inclusion and $P_{n}:\left[L\left(l_{2}, l_{2}\right), \alpha\right] \rightarrow\left[L\left(l_{2}^{n}, l_{2}^{n}\right), \alpha\right]$ be the natural projection. By Lemma 1 , since $\left\|J_{n}\right\|,\left\|P_{n}\right\| \leqq 1$ and $P_{n} J_{n}$ is the identity on $L\left(l_{2}^{n}, l_{2}^{n}\right)$ then

$$
\begin{aligned}
l\left(\left[L\left(l_{2}, l_{2}\right), \alpha\right]\right) & \geqq l\left(\left[L\left(l_{2}^{n}, l_{2}^{n}\right), \alpha\right]\right) \\
& \geqq(2 / \pi)^{2} e^{-1 / 2} \alpha(n) \underset{n \rightarrow \infty}{\longrightarrow} \infty .
\end{aligned}
$$


Let $H$ be a Hilbert space, $c_{p}(H)$ be the closure of all finite-rank operators $A: H \rightarrow H$ in the $c_{p}$ norm $\sigma_{p}$ defined by: $\sigma_{p}(A)=$ $\left[\operatorname{trace}\left(A^{*} A\right)^{p / 2}\right]^{1 / p}$ if $1 \leqq p<\infty$, and $\sigma_{\infty}(A)=\|A\|$ if $p=\infty([8])$.

COROLlaRY 2. $\quad l\left(c_{p}\left(l_{2}^{n}\right)\right) \geqq n^{|1 / p-1 / 2|} e^{-1 / 2}(2 / \pi)^{2}$ and $l\left(c_{p}\left(l_{2}\right)\right)=\infty$ if $p \neq 2$.

Proof. Taking $\alpha=\sigma_{p}$, the result follows from the fact that $\sigma_{p}(n) \geqq$ $n^{|1 / p-1 / 2|}$, Theorem 1 and Lemma 1.

Let $\left[L_{0}\left(l_{2}, l_{2}\right), \alpha\right]$ be the closure of the finite-rank operators on $l_{2}$ normed by the ideal norm $\alpha$.

If $\alpha$ is not equivalent to the Hilbert-Schmidt norm, then the following result shows that $\left[L_{0}\left(l_{2}, l_{2}\right), \alpha\right]$ does not have an unconditional Schauder decomposition into finite-diemnsional spaces if their dimensions are not sufficiently rapidly increasing.

THEOREM 2. If $p_{n} n=1,2, \cdots$, is a sequence of integers for which $\alpha(n) p_{n}^{-1 / 2} \rightarrow \infty$, then $\left[L_{0}\left(l_{2}, l_{2}\right), \alpha\right]$ does not have an unconditional Schauder decomposition into finite-dimensional spaces $E_{\imath}$ having the following property: For any $n$, there is a subset $I_{n}$ of integers for which $\left[L\left(l_{2}^{n}, l_{2}^{n}\right), \alpha\right]$ is contained in $\Sigma_{i \in I_{n}} \otimes E_{i}$ where $\operatorname{dim}\left(E_{i}\right) \leqq p_{n}$ for all $i \in I_{n}$.

Proof. Assume to the contrary $\left[L_{0}\left(l_{2}, l_{2}\right), \alpha\right]$ has such an unconditional decomposition. Fix $n$ and consider the factorization

$$
\left[L\left(l_{2}^{n}, l_{2}^{n}\right), \alpha\right] \stackrel{J_{n}}{\longrightarrow}\left[L_{0}\left(l_{2}, l_{2}\right), \alpha\right] \stackrel{P_{i}}{\longrightarrow} E_{i} \stackrel{T_{i}}{\longrightarrow}\left[\left(L_{0}\left(l_{2}, l_{2}\right), \alpha\right] \stackrel{Q_{n}}{\longrightarrow}\left[L\left(l_{2}^{n}, l_{2}^{n}\right), \alpha\right]\right.
$$

where $i \in I_{n}, J_{n}$ and $T_{i}$ are the natural inclusion operators, $P_{l}$ and $Q_{n}$ are the natural projections. Let $R_{i}=Q_{n} T_{i} P_{i} J_{n}$, then $r\left(R_{i}\right) \leqq \operatorname{dim}\left(E_{i}\right) \leqq p_{n}$ for all $i \in I_{n}$, and $\sum_{i \in I_{n}} R_{i}(x)=x$ for all $x \in L\left(l_{2}^{n}, l_{2}^{n}\right)$. Then

$$
\begin{aligned}
\sup _{ \pm, N}\left\|\sum_{i=1}^{N} \pm P_{i}\right\| & \geqq \sup _{ \pm}\left\|\sum_{i \in I_{n}} \pm P_{i}\right\| \\
& \geqq \sup _{ \pm}\left\|\sum_{i \in I_{n}} \pm R_{i}\right\| \\
& \geqq \sup _{ \pm}\left\|\sum_{i \in I_{n}} \pm \sqrt{r\left(R_{i}\right)} R_{i}\right\| p_{n}^{-1 / 2} \\
& \geqq p_{n}^{-1 / 2} l\left(\left[L\left(l_{2}^{n}, l_{2}^{n}\right), \alpha\right]\right) \\
& \geqq(2 / \pi)^{2} e^{-1 / 2} p_{n}^{-1 / 2} \alpha(n) \underset{n \rightarrow \infty}{\longrightarrow} \infty,
\end{aligned}
$$

which is a contradiction. 
Remarks. If $l(E)=\infty$, this does not necessarily imply that $E$ does not have an unconditional decomposition into finite-dimensional spaces. In fact, by [5], the space $c_{p}\left(l_{2}\right)$ for all $1<p<\infty$ has such a decomposition. Theorem 2 therefore informs us on the rapidity of growth of the dimensions of many unconditional decompositions of $c_{p}\left(l_{2}\right)$ $(p \neq 2)$ and is an answer to the question posed to this author by Professor A. Pelczyński at the June 1973 international conference on Banach spaces at Wabash, Indiana. The author learned from Professor J. Lindenstrauss that he has proved $c_{p}\left(l_{2}\right)$ imbeds in a Banach space with an unconditional basis for any $1<p<\infty$.

Finally, it should be mentioned that the condition imposed on $I_{n}$ in Theorem 2 is a very natural one, since $l_{p}$ has an unconditional basis and is isomorphically complemented in $c_{p}\left(l_{2}\right)$ hence $c_{p}\left(l_{2}\right)$ has an unconditional Schauder decomposition such that an infinite number of spaces have dimensions equal to 1 .

\section{Unconditional decompositions in $\left[L\left(l_{1}, c_{0}\right), \alpha\right]$.}

THEOREM 3. Let $\alpha$ be any ideal norm, $E=\left[L\left(l_{1}^{n}, l_{\infty}^{n}\right), \alpha\right]$. Then for any operator $B \in L\left(l_{1}^{n}, l_{\infty}^{n}\right)$

$$
e^{2} l(E)\|B\| \geqq \alpha(B) .
$$

Proof. Let $u=\sum_{i=1}^{m} A_{i} \otimes B_{i}$ be any rank-m operator mapping $E^{\prime}=\left[L\left(l_{\infty}^{n}, l_{1}^{n}\right), \alpha^{*}\right]$ to $E^{\prime}$, where $A_{i} \in E$ and $B_{i} \in E^{\prime}$. Set

$$
A_{i}\left(e_{j}\right)=\sum_{k=1}^{n} a_{i j k} f_{k}, \quad B_{i}\left(f_{j}\right)=\sum_{k=1}^{n} b_{i j k} e_{k}
$$

where $\left\{e_{k}, f_{k}\right\}_{k=1}^{n}$ is the usual biorthonormal set for $l_{1}^{n}$.

Let $A \in L\left(l_{\infty}^{n}, l_{1}^{n}\right)$ be an arbitrary non-zero operator. Define on $K=K_{E^{\prime}} \times K_{E}$ the probability measure $\mu$ by

$$
\mu(f)=\frac{2^{-4 n}}{(n !)^{2}} \sum_{\epsilon, \theta, \phi, \lambda} \sum_{n, \sigma} f\left(\left(\left[\alpha^{*}(A)\right]^{-1} h_{\theta} g_{\pi} A g_{\sigma} h_{\epsilon}\right) \times(\phi \otimes \lambda)\right)
$$

$(f \in C(K))$, where the first $\Sigma$ sums over all possible vectors $\epsilon, \theta, \phi, \lambda$ of the form $( \pm 1, \pm 1, \cdots, \pm 1)$, and the second $\Sigma$ sums over all possible permutations $\pi, \sigma$ of the set $\{1,2, \cdots, n\}$.

The operator $u$ defines a function denoted by $\langle u, \circ\rangle$ in $C(K)$ by

$$
\langle u, a \times b\rangle=\langle b, u(a)\rangle=\operatorname{trace}(b(u(a))), a \in K_{E^{\prime}}, b \in K_{E} .
$$

Then, 


$$
\begin{aligned}
\alpha^{*}(A) \mu(|\langle u, \circ\rangle|) \\
=\frac{2^{-4 n}}{(n !)^{2}} \sum_{\epsilon, \theta, \phi, \lambda} \sum_{\pi, \sigma}\left|\left\langle u\left(h_{\theta} g_{\pi} A g_{\sigma} h_{\epsilon}\right), \phi \otimes \lambda\right\rangle\right| \\
=\frac{2^{-4 n}}{(n !)^{2}} \sum \sum\left|\left\langle\left(u\left(h_{\theta} g_{\pi} A g_{\sigma} h_{\epsilon}\right)\right)(\lambda), \phi\right\rangle\right|
\end{aligned}
$$

Observe that if $v \in L\left(l_{\infty}^{n}, l_{1}^{n}\right)$, then by applying Khinchin's inequality twice it follows that

$$
2^{-2 n} \sum_{\lambda, \phi}|\langle v(\lambda), \phi\rangle| \geqq e^{-1}\left(\sum_{i, j=1}^{n}\left\langle v\left(f_{i}\right), f_{j}\right\rangle^{2}\right)^{1 / 2},
$$

and so

$$
\begin{aligned}
& e \alpha^{*}(A) \mu(|\langle u, \circ\rangle|) \\
& \geqq \frac{2^{-2 n}}{(n !)^{2}} \sum_{\epsilon, \theta} \sum_{\pi, \sigma}\left(\sum_{i, j=1}^{n}\left\langle\left(u\left(h_{\theta} g_{\pi} A g_{\sigma} h_{\epsilon}\right)\right)\left(f_{i}\right), f_{j}\right\rangle^{2}\right)^{1 / 2} \\
& \geqq(n !)^{-2} \sum_{\pi, \sigma}\left[\sum_{i, j=1}^{n}\left(\sum_{\epsilon, \theta} 2^{-2 n}\left|\left\langle\left(u\left(h_{\theta} g_{\pi} A g_{\sigma} h_{\epsilon}\right)\right)\left(f_{i}\right), f_{j}\right\rangle\right|\right)^{2}\right]^{1 / 2} \\
& \quad=(n !)^{-2} \sum_{\pi, \sigma}\left[\sum_{i, j=1}^{n}\left(\sum_{\epsilon, \theta} 2^{-2 n}\left|\sum_{k=1}^{m} b_{k i j} \operatorname{trace}\left(A_{k} h_{\theta} g_{\pi} A g_{\sigma} h_{\epsilon}\right)\right|\right)^{2}\right]^{1 / 2} .
\end{aligned}
$$

Again, by Khinchin's inequality for any $v: l_{\infty}^{n} \rightarrow l_{\infty}^{n}$

$$
2^{-n}\left|\sum_{\epsilon} \operatorname{trace}\left(v h_{\epsilon}\right)\right| \geqq e^{-1 / 2}\left(\sum_{s=1}^{n}\left\langle v\left(f_{s}\right), e_{s}\right\rangle^{2}\right)^{1 / 2},
$$

therefore

$$
\begin{aligned}
e^{1 / 2} \sum_{\epsilon, \theta} 2^{-2 n}\left|\sum_{k=1}^{m} b_{k i j} \operatorname{trace}\left(A_{k} h_{\theta} g_{\pi} A g_{\sigma} h_{\epsilon}\right)\right| \\
\geqq 2^{-n} \sum_{\theta}\left[\sum_{s=1}^{n}\left\langle\left(\sum_{k=1}^{m} b_{k i j} A_{k}\right) h_{\theta} g_{\pi} A g_{\sigma}\left(f_{s}\right), e_{s}\right\rangle^{2}\right]^{1 / 2} \\
\geqq\left[\sum_{s=1}^{n}\left(2^{-n} \sum_{\theta}\left|\left\langle h_{\theta} g_{\pi} A g_{\sigma}\left(f_{s}\right),\left(\sum_{k=1}^{m} b_{k i j} A_{k}^{\prime}\right)\left(e_{s}\right)\right\rangle\right|\right)^{2}\right]^{1 / 2},
\end{aligned}
$$

and another application of Khinchin's inequality shows that for any $x \in l_{1}^{n}, y \in l_{\infty}^{n}$

$$
\sum_{\theta} 2^{-n}\left|\left\langle h_{\theta}(x), y\right\rangle\right| \geqq e^{-1 / 2}\left(\sum_{r=1}^{n} x_{r}^{2} y_{r}^{2}\right)^{1 / 2} .
$$


So

$$
\begin{aligned}
e \sum_{\epsilon, \theta} & 2^{-2 n}\left|\sum_{k=1}^{m} b_{k i j} \operatorname{trace}\left(A_{k} h_{\theta} g_{\pi} A g_{\sigma} h_{\epsilon}\right)\right| \\
& \geqq\left[\sum_{r, s=1}^{n}\left\langle g_{\pi} A g_{\sigma}\left(f_{s}\right), f_{r}\right\rangle^{2}\left\langle\sum_{k=1}^{m} b_{k i j} A_{k}^{\prime}\left(e_{s}\right), e_{r}\right\rangle^{2}\right]^{1 / 2},
\end{aligned}
$$

and writing $A\left(f_{s}\right)=\sum_{t=1}^{n} a_{s, t} e_{t}(s=1, \cdots, n)$,

$$
\begin{aligned}
e^{2} \alpha^{*} & (A) \mu(|\langle\mu, \circ\rangle|) \\
& \geqq(n !)^{-2} \sum_{\pi, \sigma}\left[\sum_{i, j, r, s=1}^{n}\left(\sum_{k=1}^{m} b_{k i j} a_{k r s}\right)^{2} a_{\sigma(s), \pi^{-1}(r)}^{2}\right]^{1 / 2} \\
& \geqq\left[\sum_{t, j, r, s=1}^{n}\left(\sum_{k=1}^{m} b_{k i j} a_{k r s}\right)^{2}\left(\sum_{\pi, \sigma}(n !)^{-2}\left|a_{\sigma(s), \pi^{-1}(r)}\right|\right)^{2}\right]^{1 / 2} \\
& =n^{-2}\left[\sum_{i, j, r, s=1}^{n}\left(\sum_{k=1}^{m} b_{k i j} a_{k r s}\right)^{2}\right]^{1 / 2}\left(\sum_{p, q=1}^{n}\left|a_{p, q}\right|\right) \\
& \geqq n^{-2} m^{-1 / 2}\left(\sum_{p, q=1}^{n}\left|a_{p, q}\right|\right)|\operatorname{trace}(u)|
\end{aligned}
$$

where the last inequality is due to Lemma 2.

By duality it follows that for all $B \in L\left(l_{1}^{n}, l_{\infty}^{n}\right)$

$$
n^{2} e^{2} m^{1 / 2}\|B\| \mu(|\langle u, \circ\rangle|) \geqq \alpha(B)|\operatorname{trace}(u)|,
$$

hence for any sequence of operators $P_{i}: E \rightarrow E$ satisfying $\Sigma P_{i}(x)=x$ for all $x \in E$, and for any integer $N$,

$$
\begin{aligned}
\|B\| & n^{2} e^{2} \sup _{ \pm}\left\|\sum_{1}^{N} \pm \sqrt{r\left(P_{i}\right)} P_{i}\right\| \\
& =\|B\| n^{2} e^{2} \sup _{ \pm}\left\|\sum_{1}^{N} \pm \sqrt{r\left(P_{i}^{\prime}\right)} P_{i}^{\prime}\right\| \\
& =\|B\| n^{2} e^{2} \sup _{\|x\|=\left\|y^{\prime \prime}\right\|=1} \sum_{1}^{N}\left|\left\langle P_{i}^{\prime}(x), y^{\prime}\right\rangle\right| \sqrt{r\left(P_{i}^{\prime}\right)} \\
& \geqq\|B\| n^{2} e^{2} \sum_{1}^{N} \sqrt{r\left(P_{i}^{\prime}\right)} \mu\left(\left|\left\langle P_{i}^{\prime},\right\rangle\right|\right) \\
& \geqq \alpha(B) \sum_{1}^{N} \operatorname{trace}\left(P_{i}^{\prime}\right) \underset{N \rightarrow \infty}{\longrightarrow} \alpha(B) n^{2}
\end{aligned}
$$

and the Theorem is established. 
Corollary 4. If $\alpha$ is a perfect ideal norm not equivalent to the operator norm $\|\bullet\|$ for operators from $l_{1}$ to $c_{0}$, then $l\left(\left[L\left(l_{1}^{n}, l_{\infty}^{n}\right), \alpha\right]\right) \underset{n \rightarrow \infty}{\longrightarrow} \infty$ and $l\left(\left[L\left(l_{1}, c_{0}\right), \alpha\right]\right)=\infty$.

Proof. Suppose $l\left(\left[L\left(l_{1}^{n}, l_{\infty}^{n}\right), \alpha\right]\right) \leqq \lambda<\infty$ for all. $n$. Then $\|B\| \leqq$ $\alpha(B) \leqq e^{2} \lambda\|B\|$ for all compact operators $B$ from $l_{1}$ to $c_{0}$. Therefore for every operator $B \in L\left(l_{1}, c_{0}\right),\|B\| \leqq \alpha^{* *}(B) \leqq e^{2} \lambda\|B\|$.

But as $\alpha$ is perfect, $\alpha=\alpha^{* *}$, so $\alpha$ is equivalent to the operator norm $\|\circ\|$, which is a contradiction.

REMARKS. Observe that if $\alpha=\|\circ\|$ for operators from $l_{1}$ to $c_{0}$, then $L\left(l_{1}^{n}, l_{\infty}^{n}\right)$ has an unconditional basis with basis constant equal to 1 , the usual basis $f_{k} \otimes f_{j}(k, j=1, \cdots, n)$.

By duality if $\beta$ is a perfect ideal norm not equivalent to the integral norm $i_{1}\left(=\|\circ\|^{*}\right)$ for operators from $c_{0}$ to $l_{1}$, then again

$$
l\left(\left[L\left(l_{\infty}^{n}, l_{1}^{n}\right), \beta\right]\right) \underset{n \rightarrow \infty}{\longrightarrow} \infty \quad \text { and } \quad l\left(\left[L\left(c_{0}, l_{1}\right), \beta\right]\right)=\infty .
$$

As in Theorem 2, if $\beta(n)=\sup \left\{\alpha(B) /\|B\| ; B \in L\left(l_{1}^{n}, l_{\infty}^{n}\right)\right\}$ and $p_{n}$ is a sequence of integers satisfying $\beta(n) p_{n}^{-1 / 2} \underset{n \rightarrow \infty}{\longrightarrow}$, then the space of compact operators from $l_{1}$ to $c_{0}$ normed by $\alpha$ does not have an unconditional Schauder decomposition into finite-dimensional spaces $E_{i}$ with the following property: For any integer $n$ there is a subset $I_{n}$ of integers such that $L\left(l_{1}^{n}, l_{\infty}^{n}\right)$ is a subspace of $\sum_{i \in I_{n}} \otimes E_{l}$ where $\operatorname{dim}\left(E_{\imath}\right) \leqq p_{n}$ for each $i \in I_{n}$.

\section{REFERENCES}

1. D. J. H. Garling, Absolutely p-summing operators in Hilbert spaces, Studia Math., 38 (1970), 319-331.

2. Y. Gordon, On p-absolutely summing constants of Banach spaces, Israel J. Math., 7 (1969), $151-163$.

3. Y. Gordon and D. R. Lewis, Absolutely summing operators and local unconditional structures, Acta. Math., 133 (1974), 27-48.

4. Y. Gordon, D. R. Lewis and J. R. Retherford, Banach ideals of operators with applications, J. Funct. Anal., 14 (1973), 85-129.

5. S. Kwapien and A. Pelczyński, The main triangle projection in matrix spaces and its applications, Studia Math., 34 (1970), 43-68.

6. D. R. Lewis, A Banach space characterization of the Hilbert-Schmidt class, to appear.

7. J. Lindenstrauss and M. Zippin, Banach spaces with sufficiently many Boolean algebras of projections, J. Math. Anal. Appl., 25 (1969), 309-320.

8. C. A. McCarthy, $c_{p}$, Israel J. Math., 5 (1967), 249-271.

9. N. Tomczak-Jaegermann, The moduli of smoothness and convexity and the Rademacher averages of trace classes $S_{p}(1 \leqq p<\infty)$, Studia Math., 50 (1974), 163-182.

Received April 29, 1975. Supported in part by N.S.F. Grant GP-34193.

TeChNion-IsRael Institute of Technology, Haifa 


\section{PACIFIC JOURNAL OF MATHEMATICS}

\section{EDITORS}

RICHARD ARENS (Managing Editor)

University of California

Los Angeles, California 90024

\author{
R. A. Beaumont \\ University of Washington \\ Seattle, Washington 98105
}

\section{J. DugundII}

Department of Mathematics

University of Southern California

Los Angeles, California 90007

D. Gilbarg and J. Milgram

Stanford University

Stanford, California 94305

\section{ASSOCIATE EDITORS}
E. F. BECKENBACH
B. H. NeumanN
F. WoLF
K. YoshidA

\section{SUPPORTING INSTITUTIONS}

\author{
UNIVERSITY OF BRITISH COLUMBIA \\ CALIFORNIA INSTITUTE OF TECHNOLOGY \\ UNIVERSITY OF CALIFORNIA \\ MONTANA STATE UNIVERSITY \\ UNIVERSITY OF NEVADA \\ NEW MEXICO STATE UNIVERSITY \\ OREGON STATE UNIVERSITY \\ UNIVERSITY OF OREGON \\ OSAKA UNIVERSITY
}

\author{
UNIVERSITY OF SOUTHERN CALIFORNIA \\ STANFORD UNIVERSITY \\ UNIVERSITY OF TOKYO \\ UNIVERSITY OF UTAH \\ WASHINGTON STATE UNIVERSITY \\ UNIVERSITY OF WASHINGTON \\ AMERICAN MATHEMATICAL SOCIETY
}

The Supporting Institutions listed above contribute to the cost of publication of this Journal, but they are not owners or publishers and have no responsibility for its contents or policies.

Mathematical papers intended for publication in the Pacific Journal of Mathematics should be in typed form or offset-reproduced (not dittoed), double spaced with large margins. Underline Greek letters in red, German in green, and script in blue. The first paragraph or two must be capable of being used separately as a synopsis of the entire paper. Items of the bibliography should not be cited there unless absolutely necessary, in which case they must be identified by author and Journal, rather than by item number. Manuscripts, in duplicate, may be sent to any one of the four editors. Please classify according to the scheme of Math. Reviews, Index to Vol. 39. All other communications should be addressed to the managing editor, or Elaine Barth, University of California, Los Angeles, California, 90024.

100 reprints are provided free for each article, only if page charges have been substantially paid. Additional copies may be obtained at cost in multiples of 50.

The Pacific Journal of Mathematics is issued monthly as of January 1966. Regular subscription rate: $\$ 72.00$ a year (6 Vols., 12 issues). Special rate: $\$ 36.00$ a year to individual members of supporting institutions.

Subscriptions, orders for back numbers, and changes of address should be sent to Pacific Journal of Mathematics, 103 Highland Boulevard, Berkeley, California, 94708.

PUBLISHED BY PACIFIC JOURNAL OF MATHEMATICS, A NON-PROFIT CORPORATION Printed at Jerusalem Academic Press, POB 2390, Jerusalem, Israel.

\section{Copyright (C) 1975 Pacific Journal of Mathematics All Rights Reserved}




\section{Pacific Journal of Mathematics

Vol. 60, No. $2 \quad$ October, 1975

Waleed A. Al-Salam and A. Verma, A fractional Leibniz q-formula ........... 1

Robert A. Bekes, Algebraically irreducible representations of $L_{1}(G) \ldots \ldots \ldots \ldots 11$

Thomas Theodore Bowman, Construction functors for topological

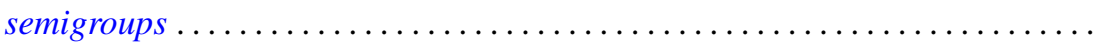

Stephen LaVern Campbell, Operator-valued inner functions analytic on the

closed disc. II .........................................

Leonard Eliezer Dor and Edward Wilfred Odell, Jr., Monotone bases in $L_{p} \ldots \ldots$.

Yukiyoshi Ebihara, Mitsuhiro Nakao and Tokumori Nanbu, On the existence of

global classical solution of initial-boundary value problem for

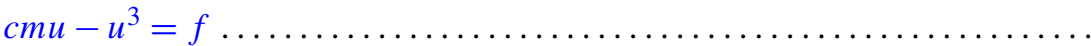

Y. Gordon, Unconditional Schauder decompositions of normed ideals of

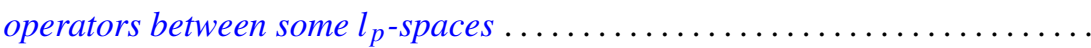

Gary Grefsrud, Oscillatory properties of solutions of certain nth order functional

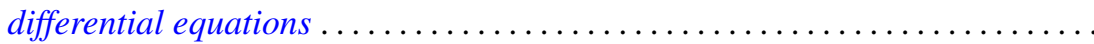

Irvin Roy Hentzel, Generalized right alternative rings ...................

Zensiro Goseki and Thomas Benny Rushing, Embeddings of shape classes of compacta in the trivial range .................................

Emil Grosswald, Brownian motion and sets of multiplicity . .

Donald LaTorre, A construction of the idempotent-separating congruences on a

bisimple orthodox semigroup .

Pjek-Hwee Lee, On subrings of rings with involution ...

Marvin David Marcus and H. Minc, On two theorems of Frobenius ...

Michael Douglas Miller, On the lattice of normal subgroups of a direct

product. .

Grattan Patrick Murphy, A metric basis characterization of Euclidean space

Roy Martin Rakestraw, A representation theorem for real convex functions ....

Louis Jackson Ratliff, Jr., On Rees localities and $H_{i}$-local rings ...

Simeon Reich, Fixed point iterations of nonexpansive mapping . .

Domenico Rosa, $B$-complete and $B_{r}$-complete topological algebras ...

Walter Roth, Uniform approximation by elements of a cone of real-valued

functions ....

Helmut R. Salzmann, Homogene kompakte projektive Ebenen

Jerrold Norman Siegel, On a space between $B H$ and $B_{\infty} \ldots$

235

Robert C. Sine, On local uniform mean convergence for Markov operators

James D. Stafney, Set approximation by lemniscates and the spectrum of an

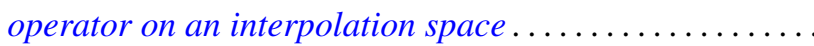

Árpád Száz, Convolution multipliers and distributions .......

Kalathoor Varadarajan, Span and stably trivial bundles ..........

Robert Breckenridge Warfield, Jr., Countably generated modules over

commutative Artinian rings....................... 